\title{
ACCURATE SOLUTION ESTIMATES FOR VECTOR DIFFERENCE EQUATIONS
}

\author{
RIGOBERTO MEDINA
}

Received 10 October 2002

\begin{abstract}
Accurate estimates for the norms of the solutions of a vector difference equation are derived. They give us stability conditions and bounds for the region of attraction of the stationary solution. Our approach is based on estimates for the powers of a constant matrix. We also discuss applications of our main results to partial reaction-diffusion difference equations and to a Volterra difference equation.
\end{abstract}

2000 Mathematics Subject Classification: 39A10.

1. Introduction. Let $\mathbb{C}^{n}$ be the set of $n$-complex vectors endowed with the Euclidean norm $\|\cdot\|$. Consider the equation

$$
x_{k+1}=A x_{k}+f_{k}\left(x_{k}\right), \quad k=0,1,2, \ldots,
$$

where $A$ is an $n \times n$ complex matrix and $f_{k}: \mathbb{C}^{n} \rightarrow \mathbb{C}^{n}$ are given functions.

A well-known result of Perron which dates back to 1929 (see [8], [12, page 270], and [10, Theorem 9.14]) states that (1.1) is asymptotically stable provided that $A$ is stable (i.e., the spectral radius $\rho(A)$ of $A$ is less than 1 ) and $f_{k}(x)=$ $\tilde{f}(x)=o(\|x\|)$. Clearly, this kind of results for the perturbed equation (1.1) is purely local. It gives no information about the size of the region of asymptotic stability and the norms of solutions.

In this paper, we derive accurate estimates for the norms of solutions. They give us stability conditions for (1.1) and bounds for the region of attraction of the stationary solution. Our approach is based on recent estimates for the powers of a constant matrix, namely, Corduneanu [3] established that for any constant matrix $A$, there exists a constant $N \geq 1$, independent of the integers $k=0,1,2, \ldots$, such that

$$
\left\|A^{k}\right\| \leq N \rho^{k}(A) \text { for } k=0,1,2, \ldots
$$

In particular, if $A=\left(a_{i j}\right)$ is a triangular constant matrix, then $N=1$. On the other hand, Gil' [5, Theorem 1.2.1] established a very sharp estimate for the powers $A^{k}$ of a constant matrix $A$. In order to establish Theorems 2.1 and 2.3, we use a method introduced by Gil' and Cheng [7] which deals with perturbed linear discrete dynamical systems. 
We also discuss the applications of our main results to a partial reactiondiffusion difference equation and to the discrete analogous of an integrodifferential equation, respectively.

2. Main results. For a positive number $r \leq \infty$, denote the ball $B_{r}=\left\{x \in \mathbb{C}^{n}\right.$ : $\|x\| \leq r\}$ and assume that there are constants $q, \mu \geq 0$ such that

$$
\left\|f_{k}(x)\right\| \leq q\|x\|+\mu \quad\left(x \in B_{r}, k=0,1,2, \ldots\right) .
$$

Assume that

$$
0<\rho(A)<1
$$

and put $\theta=N /(1-\rho(A))$.

Now, we are in a position to formulate the main result of this paper.

THEOREM 2.1. Under conditions (2.1) and (2.2), let $q \theta<1$. Then any solution $\left\{x_{k}\right\}_{k=0}^{\infty}$ of (1.1) satisfies the inequality

$$
\sup _{k=1,2, \ldots}\left\|x_{k}\right\| \leq \frac{N\left\|x_{0}\right\|+\mu \theta}{1-q \theta}
$$

provided that

$$
\left(N\left\|x_{0}\right\|+\mu \theta\right)(1-q \theta)^{-1} \leq r .
$$

Proof. By inductive arguments, it is easy to check that the unique solution $\left\{x_{k}\right\}_{k=0}^{\infty}$ of (1.1) under the initial condition $x_{0}$ is given by

$$
x_{k}=A^{k} x_{0}+\sum_{j=0}^{k-1} A^{k-j-1} f_{j}\left(x_{j}\right), \quad k=0,1,2, \ldots
$$

There are two cases to consider: $r=\infty$ and $r<\infty$.

Assuming first that $r=\infty$, hence

$$
\left\|x_{k}\right\| \leq\left\|A ^ { k } \left|\left\|\mid x_{0}\right\|+\sum_{j=0}^{k-1}\left\|A^{k-j-1}\right\|\left(q\left\|x_{j}\right\|+\mu\right), \quad k=0,1,2, \ldots\right.\right.
$$

Let $\Gamma=\sup _{k \geq 0}\left\|A^{k}\right\|$. It follows that

$$
\begin{aligned}
\left\|x_{k}\right\| \leq & \Gamma\left\|x_{0}\right\|+q \max _{i=0,1, \ldots, k-1}\left\|x_{i}\right\| \sum_{j=0}^{k-1}\left\|A^{k-j-1}\right\| \\
& +\mu \sum_{j=0}^{k-1}\left\|A^{k-j-1}\right\|, \quad k=0,1,2, \ldots
\end{aligned}
$$


By (1.2) and (2.2), we have

$$
\Gamma=\sup _{m \geq 0}\left\|A^{m}\right\| \leq \sup _{m \geq 0}\left(N \rho^{m}(A)\right)=N \sup _{m \geq 0} \rho^{m}(A)=N .
$$

Furthermore, since $0<\rho(A)<1$, we obtain

$$
\sum_{m=0}^{\infty}\left\|A^{m}\right\| \leq N \sum_{m=0}^{\infty} \rho^{m}(A)=\frac{N}{1-\rho(A)}=\theta .
$$

In view of (2.7) and the condition $q \theta<1$, it follows that

$$
\left\|x_{k}\right\| \leq N\left\|x_{0}\right\|+q \max _{i=0,1, \ldots, k-1}\left\|x_{i}\right\| \sum_{j=0}^{\infty}\left\|A^{j}\right\|+\mu \sum_{j=0}^{\infty}\left\|A^{j}\right\|, \quad k=0,1,2, \ldots
$$

Consequently,

$$
\max _{i=0,1, \ldots, k}\left\|x_{i}\right\| \leq N\left\|x_{0}\right\|+q \theta \max _{i=0,1, \ldots, k}\left\|x_{i}\right\|+\mu \theta .
$$

Hence, it follows that

$$
\begin{gathered}
\sup _{i \geq 0}\left\|x_{i}\right\|(1-q \theta) \leq N\left\|x_{0}\right\|+\mu \theta, \\
\sup _{i \geq 0}\left\|x_{i}\right\| \leq \frac{N\left\|x_{0}\right\|+\mu \theta}{1-q \theta} .
\end{gathered}
$$

Next, we will consider the case $r<\infty$.

Define the function

$$
\widetilde{f_{k}}(x)= \begin{cases}f_{k}(x), & \|x\| \leq r \\ 0, & \|x\|>r\end{cases}
$$

Since $\left\|\widetilde{f_{k}}(x)\right\| \leq q\|x\|+\mu(k=0,1,2, \ldots)$ and $x \in B_{\infty}$, then the sequence $\left\{\widetilde{x_{k}}\right\}_{k=0}^{\infty}$ defined by

$$
\begin{gathered}
\widetilde{x_{0}}=x_{0}, \\
\widetilde{x_{k+1}}=A \widetilde{x_{k}}+\widetilde{f_{k}}\left(\widetilde{x_{k}}\right), \quad k=0,1, \ldots,
\end{gathered}
$$

satisfies the inequality

$$
\sup _{k \geq 0}\left\|\widetilde{x_{k}}\right\| \leq \frac{N\left\|x_{0}\right\|+\mu \theta}{1-q \theta} \leq r
$$

provided that $\left(N\left\|x_{0}\right\|+\mu \theta\right)(1-q \theta)^{-1} \leq r$.

But $f_{k}(x)$ and $\widetilde{f}_{k}(x)$ coincide on $B_{r}$. From this, we infer that $x_{k}=\widetilde{x}_{k}$ for $k=0,1,2, \ldots$, and therefore (2.3) is satisfied, concluding the proof. 
REMARKS. (a) Under (2.1) with $\mu=0, f_{k}(0)=0$ so that $\{0\}$ is a solution of (1.1). Under condition $q \theta<1$, Theorem 2.1 asserts that the trivial solution is stable and that any initial vector $x_{0} \in B_{r}$, satisfying the condition $\left\|x_{0}\right\| \leq$ $(1-q \theta) / N$, belongs to the region of attraction.

(b) If $q=0$, then, by (2.4), every solution of (1.1) with the initial vector $x_{0}$ satisfying $N\left\|x_{0}\right\|+\mu \theta \leq r$ is bounded.

COROLlary 2.2. If $A$ is a triangular matrix and $q<1-\rho(A)$, then any solution $\left\{x_{k}\right\}_{k=0}^{\infty}$ of (1.1) satisfies

$$
\sup _{k \geq 0}\left\|x_{k}\right\| \leq \frac{(1-\rho(A))\left\|x_{0}\right\|+\mu}{1-(q+\rho(A))}
$$

provided that

$$
\left[(1-\rho(A))\left\|x_{0}\right\|+\mu\right][1-(q+\rho(A))]^{-1} \leq r .
$$

THEOREM 2.3. Suppose that $f_{k}: \mathbb{C}^{n} \rightarrow \mathbb{C}^{n}$ satisfies the condition

$$
\left\|f_{k}(x)\right\| \leq q_{k}\|x\|+\mu \quad\left(x \in B_{r}, k=0,1,2, \ldots\right)
$$

such that $\sum_{j=0}^{\infty} q_{j} \rho^{j}(A)=\beta<\infty$. If $\beta N<1$, then any solution $\left\{x_{k}\right\}_{k=0}^{\infty}$ of $(1.1)$ satisfies

$$
\sup _{k=1,2, \ldots}\left\|x_{k}\right\| \leq \frac{N\left\|x_{0}\right\|+\mu \theta}{1-\beta N}
$$

provided that $\left(N\left\|x_{0}\right\|+\mu \theta\right)(1-\beta N)^{-1} \leq r$.

By using arguments similar to those in Theorem 2.1, the result follows, thus we will omit the proof.

3. Applications. In this section, we will illustrate our main results by considering a partial difference equation and the discrete analogous of an integrodifferential equation, respectively.

We consider a simple three-level discrete reaction-diffusion equation of the form

$$
u_{i}^{(j+1)}=a u_{i-1}^{(j)}+b u_{i}^{(j)}+c u_{i+1}^{(j)}+g_{i}^{(j)}+f_{j}\left(u_{i}^{(j)}\right)
$$

defined on $\Omega=\{(i, j): i=0,1,2, \ldots, n+1 ; j=0,1,2, \ldots\}$, where $g=\left\{g_{i}^{(j)}\right\}$ is a complex function defined on $\Omega$ and $f_{j}: \mathbb{C} \rightarrow \mathbb{C}(j=0,1,2, \ldots)$ are given functions. 
Assume that the side conditions

$$
\begin{aligned}
u_{0}^{(j)} & =\delta_{j} \in \mathbb{C}, \quad j=0,1,2, \ldots, \\
u_{n+1}^{(j)}=\gamma_{j} \in \mathbb{C}, & j=0,1,2, \ldots, \\
u_{i}^{(0)}=\tau_{i} \in \mathbb{C}, & i=1,2, \ldots,
\end{aligned}
$$

are imposed, where $\tau=\operatorname{col}\left(\tau_{1}, \tau_{2}, \ldots, \tau_{n}\right) \in \mathbb{C}^{n}$. A solution of problem (3.1), (3.2) is a discrete function $u=\left\{u_{i}^{(j)}\right\}_{(i, j) \in \Omega}$ which satisfies the relations (3.1) and (3.2).

The existence and uniqueness of solutions of that problem is obvious provided the functions $f_{j}$ are well defined.

With the notation

$$
u^{(j)}=\operatorname{col}\left(u_{1}^{(j)}, u_{2}^{(j)}, \ldots, u_{n}^{(j)}\right)
$$

the sequence $\left\{u_{i}^{(j)}\right\}_{j=0}^{\infty}$ satisfies the vector equation

$$
u^{(j+1)}=A u^{(j)}+G_{j}+F_{j}\left(u^{(j)}\right), \quad j=0,1,2, \ldots,
$$

and the initial condition $u^{(0)}=\tau$, where

$$
\begin{aligned}
A & =\left[\begin{array}{cccccc}
b & c & 0 & \ldots & \ldots & 0 \\
a & b & c & 0 & \ldots & 0 \\
0 & a & b & c & \cdots & 0 \\
\vdots & \vdots & \vdots & \vdots & \vdots & \vdots \\
0 & \ldots & \ldots & 0 & a & b
\end{array}\right], \\
G_{j} & =\operatorname{col}\left(g_{1}^{(j)}, \ldots, g_{n}^{(j)}\right)+\operatorname{col}\left(a \delta_{j}, 0, \ldots, 0, c v_{j}\right), \\
F_{j}(x) & =\operatorname{col}\left(f_{j}\left(x_{1}\right), \ldots, f_{j}\left(x_{n}\right)\right), \quad x=\left(x_{1}, \ldots, x_{n}\right) .
\end{aligned}
$$

Thus, we can write the considered problem as (1.1) with $f_{j}(x)=F_{j}(x)+G_{j}$. Assume that there are nonnegative constants $q_{1}$ and $\mu_{1}$ such that

$$
\left\|F_{j}(x)\right\| \leq q_{1}\|x\|+\mu_{1} \quad\left(x \in B_{r}, j=0,1,2, \ldots\right) .
$$

In addition, assume that

$$
\mu_{2}=\sum_{j=0}^{\infty}\left\|G_{j}\right\|<\infty .
$$

Hence, condition (2.1) holds with $\mu=\mu_{1}+\mu_{2}$. 
We want to point out that if $a c>0$, then the spectral radius $\rho(A)$ is equal to $|b|+2 \sqrt{a c} \cos (\pi /(n+1))$ and when $a c<0$, then

$$
\rho(A)=\sqrt{b^{2}-4 a c \cos ^{2}\left(\frac{\pi}{n+1}\right)} .
$$

Then, as a direct consequence of Theorem 2.1, we get the following theorem.

THEOREM 3.1. Assume that

(i) $a c>0$ and $|b|+2 \sqrt{a c} \cos (\pi /(n+1))<1$, or

(ii) $a c<0$ and $b^{2}-4 a c \cos ^{2}(\pi /(n+1))<1$,

(iii) condition (2.1) holds with $\mu=\mu_{1}+\mu_{2}$.

Then, for any $x_{0}=\tau$ such that $(N\|\tau\|+\mu \theta) /\left(1-q_{1} \theta\right) \leq r$, the unique solution $x_{j}=\left\{u_{i}^{(j)}\right\}_{(i, j) \in \Omega}$ of problem (3.1), (3.2) satisfies

$$
\sup _{j=1,2, \ldots}\left|u_{i}^{(j)}\right| \leq \frac{N\|\tau\|+\mu \theta}{1-q_{1} \theta} \quad \text { for } i=0,1,2, \ldots, n+1 .
$$

REMARKS. (1) An important class of admissible perturbation functions $f_{j}$ for (3.1) is the polynomial functions. For example, if we consider

$$
f_{j}\left(x_{i}\right)=\sum_{l=0}^{p} \frac{j}{j^{2}+1} x_{i}^{\beta_{l}}+\eta_{i}, \quad j=0,1,2, \ldots, i=0,1,2, \ldots, n,
$$

where $p$ is a nonnegative integer and $\eta_{i}$ and $\beta_{l}, l=0,1,2, \ldots, p$, are nonnegative real numbers, then we infer that the requirements of Theorem 3.1 are satisfied taking $q_{1}=\sum_{l=0}^{p} r^{\beta_{l}-1}$ and $\mu_{1}=\sum_{i=0}^{n} \eta_{i}$.

Indeed, if $u^{(j)} \in B_{r}$, then

$$
\begin{aligned}
\left|f_{j}\left(u_{i}^{(j)}\right)\right| & \leq \sum_{l=0}^{p}\left|u_{i}^{(j)}\right|\left|u_{i}^{(j)}\right|^{\beta_{l}-1}+\eta_{i} \\
& \leq \sum_{l=0}^{p} r^{\beta_{l}-1}\left|u_{i}^{(j)}\right|+\eta_{i} \\
& \leq\left(\sum_{l=0}^{p} r^{\beta_{l}-1}\right)\left|u_{i}^{(j)}\right|+\eta_{i} .
\end{aligned}
$$

Hence,

$$
\begin{aligned}
\left\|F_{j}\left(u^{(j)}\right)\right\| & =\left\|\left(f_{j}\left(u_{1}^{(j)}\right), \ldots, f_{j}\left(u_{n}^{(j)}\right)\right)\right\| \\
& \leq\left(\sum_{l=0}^{p} r^{\beta_{l}-1}\right)\left|u_{1}^{(j)}\right|+\eta_{1}+\cdots+\left(\sum_{l=0}^{p} r^{\beta_{l}-1}\right)\left|u_{n}^{(j)}\right|+\eta_{n} \\
& \leq\left(\sum_{l=0}^{p} r^{\beta_{l}-1}\right)\left\|u^{(j)}\right\|+\mu_{1} .
\end{aligned}
$$


(2) We want to point out that Theorem 3.1 compares favorably with [11, Theorems 1 and 2] and [2, Theorems 1-4]. On the other hand, Theorems 2.1 and 2.3 are comparable with the main results in [7]. However, our results, though different in their approach in general, have very little overlapping with those in $[2,7,11]$. Other important related results can be found in [1, pages 237-245], [6], [4, pages 196-204], and [9, pages 102-104].

We now consider the analogous of the integrodifferential equation

$$
\frac{d x}{d t}=B x(t)+\int_{0}^{t} f(s, x(s)) d s, \quad t \geq 0
$$

Consider the discrete equation

$$
x_{k+1}=A x_{k}+\sum_{j=0}^{k} f_{j}\left(x_{j}\right), \quad k \geq 0,
$$

where $A$ is an $n \times n$ complex matrix and $f_{j}: \mathbb{C}^{n} \rightarrow \mathbb{C}^{n}(j=0,1,2, \ldots)$ are given functions, satisfying

$$
\left\|f_{j}(x)\right\| \leq q_{j}\|x\|+\mu \quad\left(x \in B_{r}, j=0,1,2, \ldots\right) .
$$

Define

$$
S(A)=\sum_{k=0}^{\infty} k \rho^{k}(A), \quad \psi(A ; f)=\sum_{k=0}^{\infty} \rho^{k}(A)\left(\sum_{i=0}^{k} q_{i}\right) .
$$

We are now in a position to establish the next theorem.

THEOREM 3.2. Under condition (3.15), assume that

$$
S(A)<\infty, \quad N \psi(A ; f)<1 .
$$

Then any solution $\left\{x_{k}\right\}_{k=0}^{\infty}$ of (3.14) satisfies the inequality

$$
\sup _{k=1,2, \ldots}\left\|x_{k}\right\| \leq \frac{N\left\|x_{0}\right\|+\mu N S(A)}{1-N \psi(A ; f)}
$$

provided that

$$
\left(N\left\|x_{0}\right\|+\mu N S(A)\right)(1-N \psi(A ; f))^{-1} \leq r .
$$

Proof. The variation of parameters formula yields

$$
x_{k}=A^{k} x_{0}+\sum_{j=0}^{k-1} A^{k-j-1}\left(\sum_{i=0}^{j} f_{i}\left(x_{i}\right)\right), \quad k=0,1,2, \ldots
$$

There are two cases to consider: $r=\infty$ and $r<\infty$. 
Assuming first that (3.15) is valid for $r=\infty$, hence

$$
\left\|x_{k}\right\| \leq\left\|A_{k}\right\|\left\|x_{0}\right\|+\sum_{j=0}^{k-1}\left\|A^{k-j-1}\right\|\left(\sum_{i=0}^{j}\left(q_{i}\left\|x_{i}\right\|+\mu\right)\right), \quad k=0,1,2, \ldots
$$

Thus, following the lines of the proof of Theorem 2.3, the result follows.

ACKNOWLEDGMENT. This research was supported by FONDECYT Chile under Grant 1.030.460.

\section{REFERENCES}

[1] R. P. Agarwal, Difference Equations and Inequalities. Theory, Methods, and Applications, Monographs and Textbooks in Pure and Applied Mathematics, vol. 155, Marcel Dekker, New York, 1992.

[2] S. S. Cheng and J.-Y. Lin, Stability criteria for a discrete reaction-diffusion equation, Far East J. Math. Sci. 6 (1998), no. 3, 425-435.

[3] A. Corduneanu, The asymptotic behaviour of the solution of some difference equations, Bul. Inst. Politeh. Iaşi. Secț. I. Mat. Mec. Teor. Fiz. 39(43) (1993), no. 1$4,45-52$.

[4] S. N. Elaydi, An Introduction to Difference Equations, 2nd ed., Undergraduate Texts in Mathematics, Springer-Verlag, New York, 1999.

[5] M. I. Gil', Stability of Finite- and Infinite-Dimensional Systems, The Kluwer International Series in Engineering and Computer Science, vol. 455, Kluwer Academic Publishers, Massachusetts, 1998.

[6] M. I. Gil' and S. S. Cheng, Stability of a time discrete perturbed dynamical systems with delay, Discrete Dyn. Nat. Soc. 3 (1999), no. 1, 57-63.

[7] _ Estimates for the norms of solutions of a perturbed linear dynamical system, Proc. Natl. Sci. Counc. ROC (A) 24 (2000), no. 2, 95-98.

[8] S. P. Gordon, On converses to the stability theorems for difference equations, SIAM J. Control 10 (1972), 76-81.

[9] V. Lakshmikantham and D. Trigiante, Theory of Difference Equations. Numerical Methods and Applications, Mathematics in Science and Engineering, vol. 181, Academic Press, Massachusetts, 1988.

[10] J. P. LaSalle, The Stability of Dynamical Systems, Regional Conference Series in Applied Mathematics, Society for Industrial and Applied Mathematics, Pennsylvania, 1976.

[11] R. Medina and S. S. Cheng, The asymptotic behavior of the solutions of a discrete reaction-diffusion equation, Comput. Math. Appl. 43 (2002), no. 8-9, 917925.

[12] J. M. Ortega, Stability of difference equations and convergence of iterative processes, SIAM J. Numer. Anal. 10 (1973), no. 2, 268-282.

Rigoberto Medina: Departamento de Ciencias Exactas, Universidad de Los Lagos, Casilla 933, Osorno, Chile

E-mail address: rmedina@u1agos.c1 


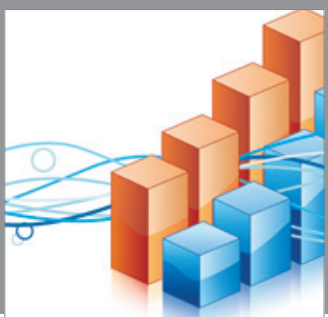

Advances in

Operations Research

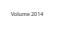

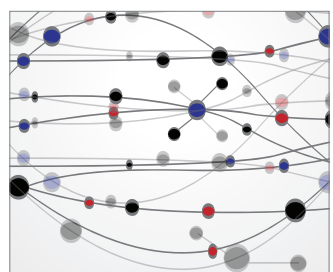

\section{The Scientific} World Journal
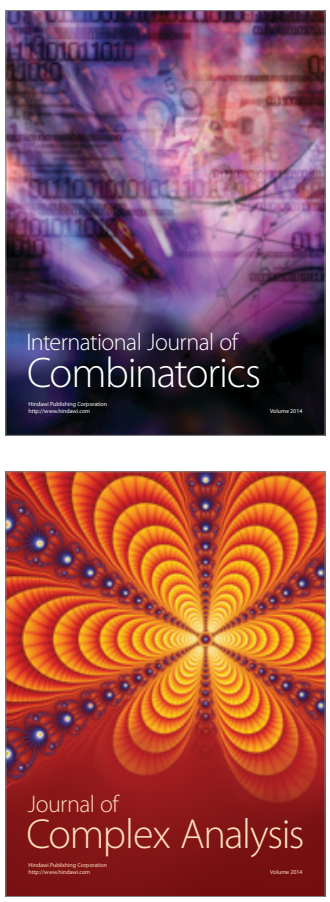

International Journal of

Mathematics and

Mathematical

Sciences
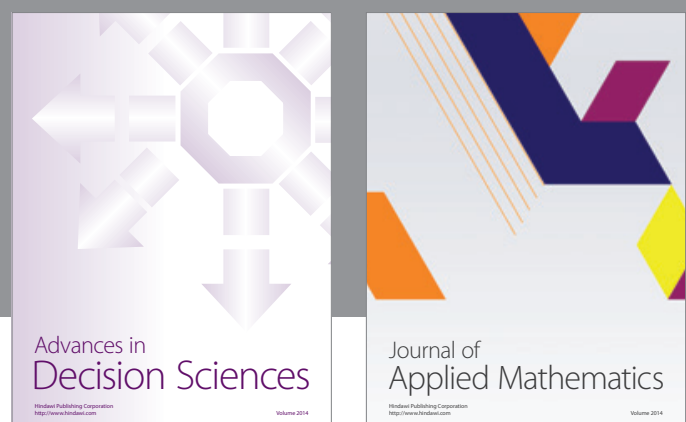

Journal of

Applied Mathematics
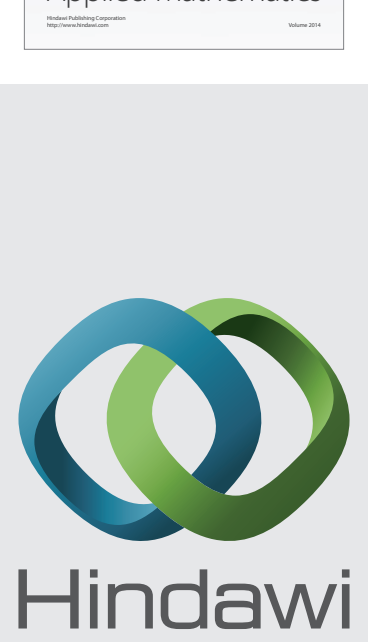

Submit your manuscripts at http://www.hindawi.com
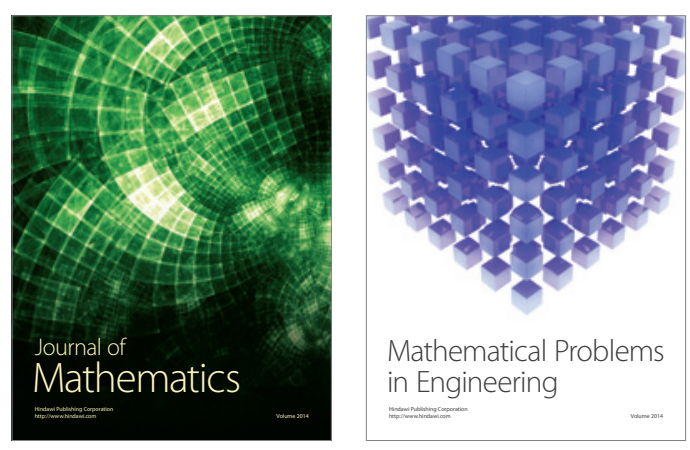

Mathematical Problems in Engineering
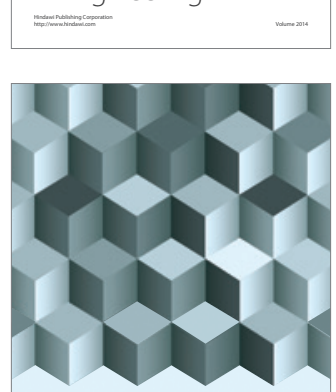

Journal of

Function Spaces
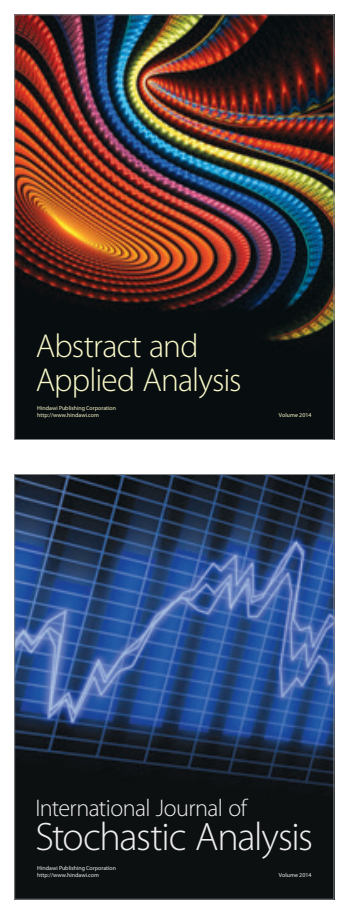

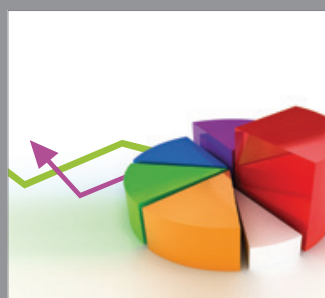

ournal of

Probability and Statistics

Promensencen
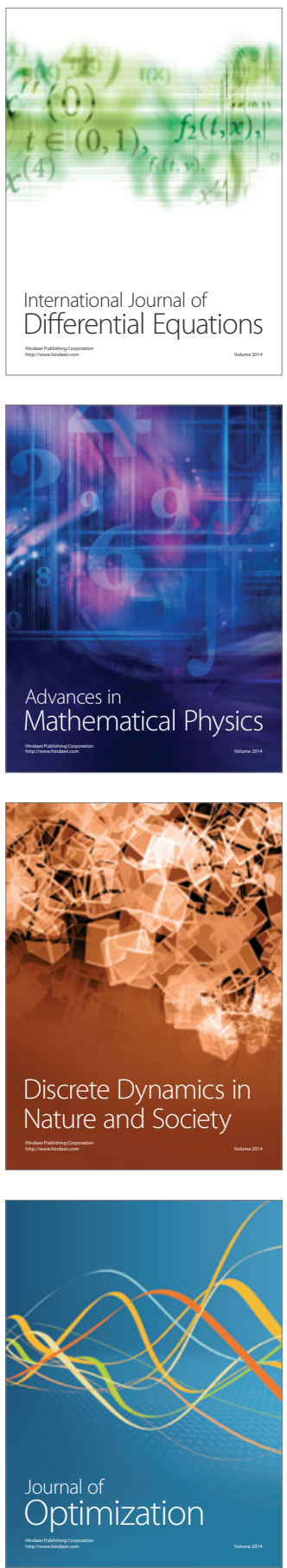